\title{
Estimation of Magnetic Field Growth and Construction of Adaptive Mesh in Corner Domain for the Magnetostatic Problem in Three-Dimensional Space
}

\author{
Eugene Perepelkin ${ }^{1 \star}$ and Aleksandr Tarelkin ${ }^{1 \star \star}$ \\ ${ }^{1}$ Lomonosov Moscow State University, GSP-1, Leninskie Gory, Moscow, 119991, Russian Federation
}

\begin{abstract}
A magnetostatics problem arises when searching for the distribution of the magnetic field generated by magnet systems of many physics research facilities, e.g., accelerators. The domain in which the boundary-value problem is solved often has a piecewise smooth boundary. In this case, numerical calculations of the problem require consideration of the solution behavior in the corner domain. In this work we obtained an upper estimation of the magnetic field growth using integral formulation of the magnetostatic problem and propose a method for condensing the differential mesh near the corner domain of the vacuum in the three-dimensional space based on this estimation.
\end{abstract}

\section{Introduction}

Many physics research facilities use magnetic systems of various configurations, e.g., a system of spectrometric magnets. It is very important to know with a good accuracy the distribution of the magnetic field generated by this system. The problem is actually reduced to the formulation of a magnetostatics problem of finding the distribution of the magnetic field generated by the magnetic system under consideration. Since the magnetic system has a complicated configuration, the solution to the problem is usually sought using numerical methods. This paper discusses the case when the domain over which a boundary value magnetostatics problem is solved has piecewise smooth boundary. In this case, the solution to the problem or the derivative solutions can have a singularity, thus the numerical search for the solution requires the use of special methods.

\section{Integral formulation of the magnetostatic problem}

The integral formulation allows the magnetic field to be represented as

$$
\vec{H}(s)=\vec{H}_{C}(s)-\nabla_{s} \int_{\Omega_{f}}\left(\vec{M}(p), \nabla_{p} \psi(s, p)\right) d v_{p},
$$

where $\vec{H}_{C}$ is the field from the current sources, $\vec{M}$ is the ferromagnetic magnetization vector, the function $\Psi(s, p)$ is equal to $1 / 4 \pi r_{s p}$ or $1 / 2 \pi \cdot \ln r_{s p}$ for the three-dimensional and the two-dimensional case respectively, and $\Omega_{f}$ is the ferromagnetic domain. The magnetization vector is defined as $\vec{M}=$

${ }^{\star}$ e-mail: pevgeny@mail.ru

$\star \star$ e-mail: tarelkin.aleksandr@physics.msu.ru 
$\mu_{0} \chi(H) \vec{H}=\mu_{0}(\mu(H)-1) \vec{H}$, where $\mu_{0}$ is a constant, $\chi(H)$ is the magnetic susceptibility, and $\mu(H)$ is the permeability of the ferromagnetic.

\section{Estimation of magnetic field growth}

The following theorem characterizes the magnetic field growth in the 3D space (the estimation for the 2D space was obtained in [1]):

\section{Theorem}

If the permeability function of the ferromagnetic has the representation $\mu(H)=1+A / H-B / H^{2}$ when $H \rightarrow \infty$, then the magnetic field in the corner domain outside a ferromagnetic satisfies the condition

$$
H(s) \leq C_{0} \ln \frac{1}{r_{s}}+w(s),
$$

where $C_{0}$ is a constant, $w(s)$ is a bounded function, and $r_{s}$ is the distance to the corner.

\section{Proof}

At high fields $(H \rightarrow \infty)$, the representation $\mu(H)=1+A / H-B / H^{2}$ when $H \rightarrow \infty$ is valid, where $A$ and $B$ are positive constants. Consequently, at $H \rightarrow \infty, M=|\vec{M}|$ is limited by a constant $M_{0}=\mu_{0} A$.

From (1) we obtain $\vec{H}(s)=\vec{H}_{C}(s)-\nabla_{s} \int_{\Omega_{f}}\left(\vec{M}(p), \vec{r}_{s p} / r_{s p}^{3}\right) d v_{p}$. Here the first term doesn't contribute directly to the effect of significant growth of the magnetic field near the corner point, and we therefore estimate the second term

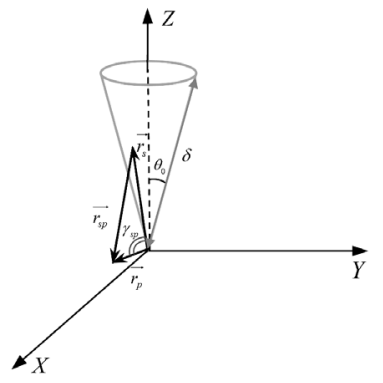

Figure 1. The conical volume

$$
\begin{gathered}
\left|\nabla_{s} \int_{\Omega_{f}}\left(\vec{M}(p), \frac{\vec{r}_{s p}}{r_{s p}^{3}}\right) d v_{p}\right| \leq\left|\int_{\Omega_{f}} \frac{\partial}{\partial x_{s}} \frac{M^{(x)}(p) \bar{x}+M^{(y)}(p) \bar{y}+M^{(z)}(p) \bar{z}}{\bar{x}^{2}+\bar{y}^{2}+\bar{z}^{2}} d v_{p}\right|+ \\
+\left|\int_{\Omega_{f}} \frac{\partial}{\partial y_{s}} \frac{M^{(x)}(p) \bar{x}+M^{(y)}(p) \bar{y}+M^{(z)}(p) \bar{z}}{\bar{x}^{2}+\bar{y}^{2}+\bar{z}^{2}} d v_{p}\right|+\left|\int_{\Omega_{f}} \frac{\partial}{\partial z_{s}} \frac{M^{(x)}(p) \bar{x}+M^{(y)}(p) \bar{y}+M^{(z)}(p) \bar{z}}{\bar{x}^{2}+\bar{y}^{2}+\bar{z}^{2}} d v_{p}\right| \leq \\
\leq 6 M_{0} \int_{\Omega_{f}} \frac{1}{r_{s p}^{3}} d v_{p},
\end{gathered}
$$

where $\bar{x}=x_{s}-x_{p}, \bar{y}=y_{s}-y_{p}$ and $\bar{z}=z_{s}-z_{p}$. We calculate the integral

$$
\int_{\Omega_{f}} \frac{1}{r_{s p}^{3}} d v_{p}=\int_{v_{\delta}} \frac{1}{r_{s p}^{3}} d v_{p}+\int_{\Omega_{f} \backslash v_{\delta}} \frac{1}{r_{s p}^{3}} d v_{p},
$$

where $v_{\delta}=\Omega_{f} \cap S_{\delta}(Q)$ is the conical volume $v_{\delta}$ at the corner point (see Figure 1).

Let us consider the integral over the domain $v_{\delta}$

$$
\int_{\Omega_{f}} \frac{1}{r_{s p}^{3}} d v_{p}=\int_{0}^{\varphi_{0}} d \varphi_{p} \int_{0}^{\theta_{0}} \sin \theta_{p} d \theta_{p} \int_{0}^{\delta} \frac{r_{p}^{2}}{r_{s p}^{3}} d r_{p}=\int_{0}^{\varphi_{0}} d \varphi_{p} \int_{0}^{\theta_{0}} \sin \theta_{p} d \theta_{p} \int_{0}^{\delta} \frac{r_{p}^{2}}{\left(r_{p}^{2}+r_{s}^{2}-2 r_{p} r_{s} \cos \gamma_{s p}\right)^{3 / 2}} d r_{p}=
$$




$$
=\int_{0}^{\varphi_{0}} d \varphi_{p} \int_{0}^{\theta_{0}} \sin \theta_{p} d \theta_{p}\left(\int_{0}^{1} \frac{t^{2} d t}{\left(1+t^{2}-2 t \cos \gamma_{s p}\right)^{3 / 2}}+\int_{1}^{\delta / r_{s}} \frac{t^{2} d t}{\left(1+t^{2}-2 t \cos \gamma_{s p}\right)^{3 / 2}}\right),
$$

$t=r_{p} / r_{s}$ and $\cos \gamma_{s p}=\sin \theta_{p} \sin \theta_{s} \cos \left(\varphi_{p}-\varphi_{s}\right)+\cos \theta_{p} \cos \theta_{s}$. Then we use the expression for the generating function

$$
\frac{1}{\sqrt{1+t^{2}-2 t \cos \varphi_{s p}}}=\left\{\begin{array}{l}
\sum_{m=0}^{+\infty} P_{m}\left(\cos \varphi_{s p}\right) t^{m}, \quad|t|<1, \\
\sum_{m=0}^{+\infty} P_{m}\left(\cos \varphi_{s p}\right) t^{-m-1}, \quad|t|<1,
\end{array}\right.
$$

and obtain

$$
\begin{gathered}
\int_{\Omega_{f}} \frac{1}{r_{s p}^{3}} d v_{p}=\int_{0}^{\varphi_{0}} d \varphi_{p} \int_{0}^{\theta_{0}} \sin \theta_{p} d \theta_{p}\left[\int_{0}^{1} t^{2} d t \sum_{m, n, k=0}^{+\infty} P_{m} P_{n} P_{k} t^{m+n+k}+\int_{1}^{\delta / r_{s}} t^{2} d t \sum_{m, n, k=0}^{+\infty} P_{m} P_{n} P_{k} t^{-(m+n+k+3)}\right]= \\
=\int_{0}^{\varphi_{0}} d \varphi_{p} \int_{0}^{\theta_{0}} \sin \theta_{p} d \theta_{p}\left[\left.\sum_{m, n, k=0}^{+\infty} P_{m} P_{n} P_{k} \frac{t^{m+n+k+3}}{m+n+k+3}\right|_{0} ^{1}+\left.\sum_{m+n+k \neq 0}^{+\infty} P_{m} P_{n} P_{k} \frac{t^{-(m+n+k)}}{-(m+n+k)}\right|_{1} ^{\delta / r_{s}}\right]+ \\
+\left.\int_{0}^{\varphi_{0}} d \varphi_{p} \int_{0}^{\theta_{0}} \sin \theta_{p} d \theta_{p} P_{0}^{2} \ln t\right|_{1} ^{\delta / r_{s}}=\omega_{0} \ln \frac{\delta}{r_{s}}+\sum_{m, n, k=0}^{+\infty} \frac{\alpha_{m, n, k}}{m+n+k+3}+\sum_{m+n+k \neq 0}^{+\infty} \frac{\alpha_{m, n, k}}{m+n+k}\left[1-\left(\frac{r_{s}}{\delta}\right)^{m+n+k}\right]= \\
=C_{1} \ln \frac{1}{r_{s}}+w_{1}(s),
\end{gathered}
$$

where $\alpha_{m, n, k}=\int_{0}^{\varphi_{0}} d \varphi_{p} \int_{0}^{\theta_{0}} \sin \theta_{p} P_{m}\left(\cos \gamma_{s p}\right) P_{n}\left(\cos \gamma_{s p}\right) P_{k}\left(\cos \gamma_{s p}\right) d \theta_{p}, C_{1}$ is a constant, and $w_{1}(s)$ is a bounded function. Thus, the validity of expression (2) is ascertained.

\section{Remark}

The estimation of field growth given above was obtained for an arbitrary form of the ferromagnetic. It is easy to select the constants $\delta, \theta_{0}$ and $\varphi_{0}$ for defining any model geometry.

\section{Method of mesh condensing in the corner domain}

Based on the estimation obtained we propose to condense the mesh approaching the corner point. Let us define the consistent step of the magnetic field value change as $\Delta h=H_{0} /\left(C_{1} N\right)$, where $H_{0}$ is the approximate maximal value of the magnetic field in the domain under consideration (it can be obtained from pre-simulation with using uniform mesh), $C_{1}$ is a constant and $N$ is the number of mesh nodes in a linear dimension. The main idea of the mesh construction is that each step of the mesh $\Delta l_{i}$ corresponds with one and the same $\Delta h$ [2]. 


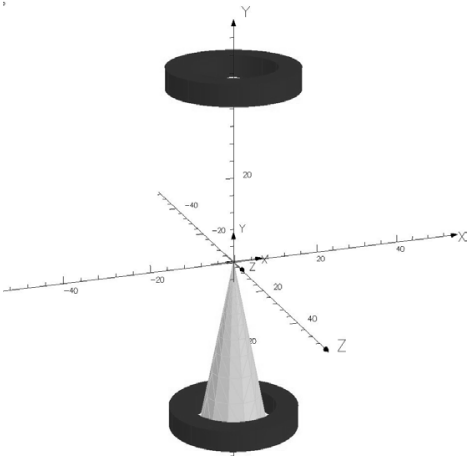

Figure 2. Model geometry

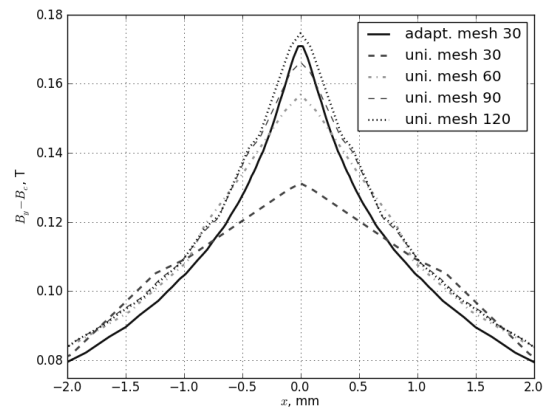

Figure 3. $B_{y}-B_{\text {coils }}$ distribution along the line for different meshes

\section{Calculation of the magnetic field in the corner domain}

We present numerical calculations of a magnet system using the obtained method. We consider the magnet system depicted in Figure 2. This is a conical ferromagnetic placed between two identical coils. A boundary-value problem corresponding to the formulation of the magnetostatic problem was solved in the domain under consideration. The efficiency of the differential mesh condensing method described in Section 4 was estimated by the following calculations: the solution of the boundary value problem was found on the sequence of uniform meshes $(30 k) \times(30 k) \times(30 k)$, where $k=1,2,3,4$. Then the same problem was calculated on a $30 \times 30 \times 30$ mesh, but the mesh spacing was chosen using the mesh condensing method. The number of node points in the domain was the same as in the case when $k=1$ for a uniform mesh, and only their distribution changed. Then all the calculated results were compared in order to assess how many nodes are needed by the uniform mesh to provide a result similar to the result obtained using the adaptive mesh. Figure 3 shows the distribution $B_{y}-B_{\text {coils }}$, which is the ferromagnetic field without contribution from coils, along the $x$-directed straight line, which starts at $x=-2, y=0.1, z=0$ and finishes at $x=2$, for different meshes. It is evident from Figure 3 that the adaptive mesh with 30 nodes in linear dimension give a comparable result to a uniform mesh with 120 nodes. Thus, it follows that the proposed constructing mesh method in the corner domain is worth using and yields results comparable in accuracy with the results obtained on meshes with the number of nodes in each axis three to four times larger than in the initial mesh.

\section{Conclusion}

1. The upper estimate for the magnetic field growth $\vec{H}(s)$ in the corner domain $H(s) \leq C_{0} \ln \frac{1}{r_{s}}+$ $w(s)$, where $C_{0}$ is a constant, $w(s)$ is a bounded function, and $r_{s}$ is the distance to the corner, is asymptotically obtained for the case of $\mu(H) \rightarrow 1$ when $H \rightarrow \infty$.

2. The condensing mesh method in the corner domain has been proposed, which allows one to reduce the number of mesh nodes at the numerical simulation of the magnetostetic problem.

\section{References}

[1] E.E. Perepelkin, R.V. Polyakova, A.D. Kovalenko, P.N. Sysoev, M.B. Sadovnikova, A.A. Tarelkin, and I.P. Yudin, RUDN Journal of MIPh. 25, 3, 253-265 (2017)

[2] E.E. Perepelkin, R.V. Polyakova, A.D. Kovalenko, L.A. Nyanina, P.N. Sysoev, M.B. Sadovnikova, and I.P. Yudin, Computer Technologies in Physics 13, 6, 782-786 (2016) 